\title{
The Effect of Finite Spatial Resolution on the Measurement of Cardiac Phantom Wall Thickness in Single Photon Emission Computerized Tomographic Imaging
}

\author{
Nasser Ballani Geoff Dougherty \\ Department of Radiologic Sciences, Faculty of Allied Health Sciences, Kuwait University and \\ Department of Nuclear Medicine, Hussein Makey Juma'a Center for Specialized Surgery, Ministry of Health, Kuwait
}

\section{Key Words}

Myocardial wall thickness · Single photon emission tomography - System resolution - Partial volume effect particularly useful for small separations (e.g. at end systole and/or towards the apex of the heart) of walls that are narrow compared to the PSF of the imaging system.

Copyright $\odot 2004$ S. Karger AG, Basel

\section{Introduction}

The limited spatial resolution of imaging systems causes difficulties in the measurement of the thickness and brightness of thin structures. Convolution of an object during imaging by the point spread function (PSF) of the imaging system causes the image to be wider and less bright than the object, and the effect is increasingly significant for object sizes smaller than twice the width of the PSF [1]. The phenomenon is exacerbated in digital systems due to the inherent finite sampling, especially when a coarse raster is used such as in nuclear medicine imaging; the resulting degradation is often referred to as the partial volume effect.

This effect was reported for computed tomography (CT) imaging systems in attempts to measure the thickness of the vertebral shell [2-8], and confirmed in myocardial studies using single photon emission tomography

\section{KARGER \\ Fax +4161306 1234 \\ E-Mail karger@karger.ch \\ www. karger.com

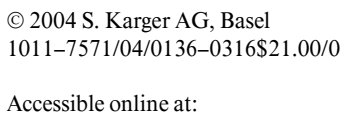

Dr. Nasser Ballani

Department of Radiologic Sciences, Faculty of Allied Health Sciences Kuwait University, PO Box 31740

90807 Sulaibikhat (Kuwait)

Tel. +965 4820833, Fax +965 4833662, E-Mail nasser@hsc.kuniv.edu.kw 
(SPECT) [1, 9-13]. Simulations of rectangular and Gaussian profiles imaged with CT systems for various fields of view (FoVs) and reconstruction kernels have clarified the role of the factors causing blurring in a digital imaging system [14].

The small, but clinically significant, variations in myocardial wall thickness during the cardiac cycle affect the evaluation of myocardial ischaemia and viability. Their accurate determination from SPECT images is difficult because of the inherently low spatial resolution and high levels of quantum noise, and because of cardiac motion that results in considerable blur even in gated images. The resolution of current clinical SPECT systems ranges from 8 to $12 \mathrm{~mm}$. Myocardial wall thicknesses vary even amongst normal subjects [15]. Typically the left ventricular wall is about $10 \mathrm{~mm}$ thick [16] at end diastole, and thickens to about $14 \mathrm{~mm}$ [17] at end systole. Thickening of less than $2 \mathrm{~mm}$ is considered abnormal [18].

The aim of this work was to devise and validate a method that would accurately measure the thickness of narrow walls even when they are in close proximity as exemplified in a SPECT image of a short-axis slice of the heart by fitting the profile of the walls to an envelope of two Gaussian functions and then deconvolving the resulting estimates with the system PSF. This avoids having to detect the endocardial and epicardial edges [19-21] or having to fit to a single Gaussian function [13, 22].

\section{Materials and Methods}

\section{Determination of the Spatial Resolution of the Clinical SPECT}

System

A microhaematocrit capillary tube of diameter $0.6 \mathrm{~mm}$ and length $75 \mathrm{~mm}$ was filled with $36 \mathrm{MBq}$ of technetium- $99 \mathrm{~m}$ pertechnetate. In order to mimic the cardiac position, the tube was fixed along the detector-head axis at the centre of a cylindrically shaped phantom of diameter $216 \mathrm{~mm}$, which was then filled with water. A SPECT system, with a GE MP dual-head gamma camera and a high-resolution collimator, was used to reconstruct images on a $128 \times 128$ matrix with a FoV of $520 \times 380 \mathrm{~mm}$ (pixel size of $\sim 4 \mathrm{~mm}$ ): the radius of rotation was $250 \mathrm{~mm}$. Thirty-two views were acquired over $180^{\circ} \mathrm{C}$ with 20 s per projection. A $20 \%$ symmetric window centred on $140 \mathrm{keV}$ of technetium-99m was used to improve sensitivity. Using a Genie processor, reconstructions of transaxial slices were obtained using a ramp-Hanning filter with a cut-off of 0.8 cycles $\mathrm{cm}^{-1}$. The total count per transaxial slice was about $5 \times 10^{4}$ with a maximum count per pixel of about $10^{3}$. A horizontal profile passing through the line activity was considered to characterize the spatial resolution, and was used in the subsequent simulations.

\section{Simulation Studies}

For simplicity, and consistent with other simulations [9, 18, 23], we assumed a linear model and used a one-dimensional Gaussian to simulate resolution degradation. This can be generalized to three dimensions, and to the case of position-dependent resolution, by appropriate convolution of the three-dimensional source distribution with the three-dimensional resolution function [24].

The profile across each myocardial wall was represented by a rectangular function. In order to investigate a range of conditions mimicking myocardial perfusion imaging studies, we used wall thicknesses and separations of 1-9 pixels symmetrically placed in a series of 64 pixels. These profiles were then convolved with the experimental profile of the spatial resolution, including noise, to produce output profiles (i.e., simulated profiles through the myocardium), whose peak heights and widths (full widths at half maximum height, FWHMs) were measured. Whether convolution was performed directly in the spatial domain or by multiplication in the frequency domain, the shapes of the output profiles were identical. The use of discrete convolution with the measured spatial resolution differed somewhat from the blurring process within the SPECT system, where the blurring was continuous and sampling occurred after detection. However, this was unlikely to cause any significant difference between the simulations and true acquisitions, except at a pixel width of one where undersampling could be an important issue.

The wall thickness (taken as FWHM) was then estimated from each profile. Each profile was considered to be the envelope of two Gaussians, even when the two Gaussians could not be resolved properly. The Gaussian fitting was performed using the Levenberg-Marquardt least squares algorithm. Where two peaks were seen, their positions were taken as the starting points for the fitting. If only a single peak could be seen, then points slightly to the right and left of the peak were used as starting points but the precise positions were not critical. The widths of the Gaussians were not constrained to be equal. The fitting algorithm allowed the widths and positions of the two Gaussians to vary to fit the data. For the cases where the valley between the peaks was greater than $50 \%$ of the peak height, the FWHM was measured directly from the profiles.

\section{Hot Ring Phantom}

A vial of $65 \mathrm{~mm}$ diameter was filled to a depth of $4 \mathrm{~mm}$ with $19 \mathrm{MBq}$ of technetium- $99 \mathrm{~m}$ pertechnetate. A lead disc of $32 \mathrm{~mm}$ diameter was placed in contact with the bottom of the vial, and moved manually to produce a series of planar images, examples of which are shown in figure 1. Planar images were acquired on a $256 \times$ 256 matrix (pixel size of $\sim 2 \mathrm{~mm}$ ) with a system resolution (FWHM) of $8.18 \pm 0.45 \mathrm{~mm}$. The right-hand image had walls of roughly equal thickness $(17.0 \pm 0.3$ and $16.0 \pm 0.3 \mathrm{~mm})$, and the left-hand image had walls of unequal thickness $(25.0 \pm 0.3$ and $8.0 \pm 0.3 \mathrm{~mm})$ (all uncertainties represent the standard deviation obtained from five repeated measurements). Profiles were drawn through the centre of each image, and the wall thicknesses estimated by fitting a combination of two Gaussians to each.

\section{Cardiac Phantom}

A cardiac insert mounted in an elliptical phantom ECT (Data Spectrum Corp., Hillsborough, N.C., USA) was used to produce reconstructed images on a $128 \times 128$ matrix (pixel size of $\sim 4 \mathrm{~mm}$ ) that simulated different slices obtained in myocardial $180^{\circ} \mathrm{C} 32-$ view SPECT imaging. The cardiac insert, which had an average wall thickness of $10.0 \pm 0.2 \mathrm{~mm}$, was filled with a uniform technetium$99 \mathrm{~m}$ activity with a count rate of $1.5 \times 10^{3}$ counts s$^{-1}$. Four profiles passing through the walls at 8 cardiac sections (anteroseptal/inferolateral, anterior/inferior, septal/lateral, and anterolateral/inferosep- 
Fig. 1. Planar images of the hot ring phantom. A lead disc was positioned to simulate unequal walls (left) and roughly equal walls (right).
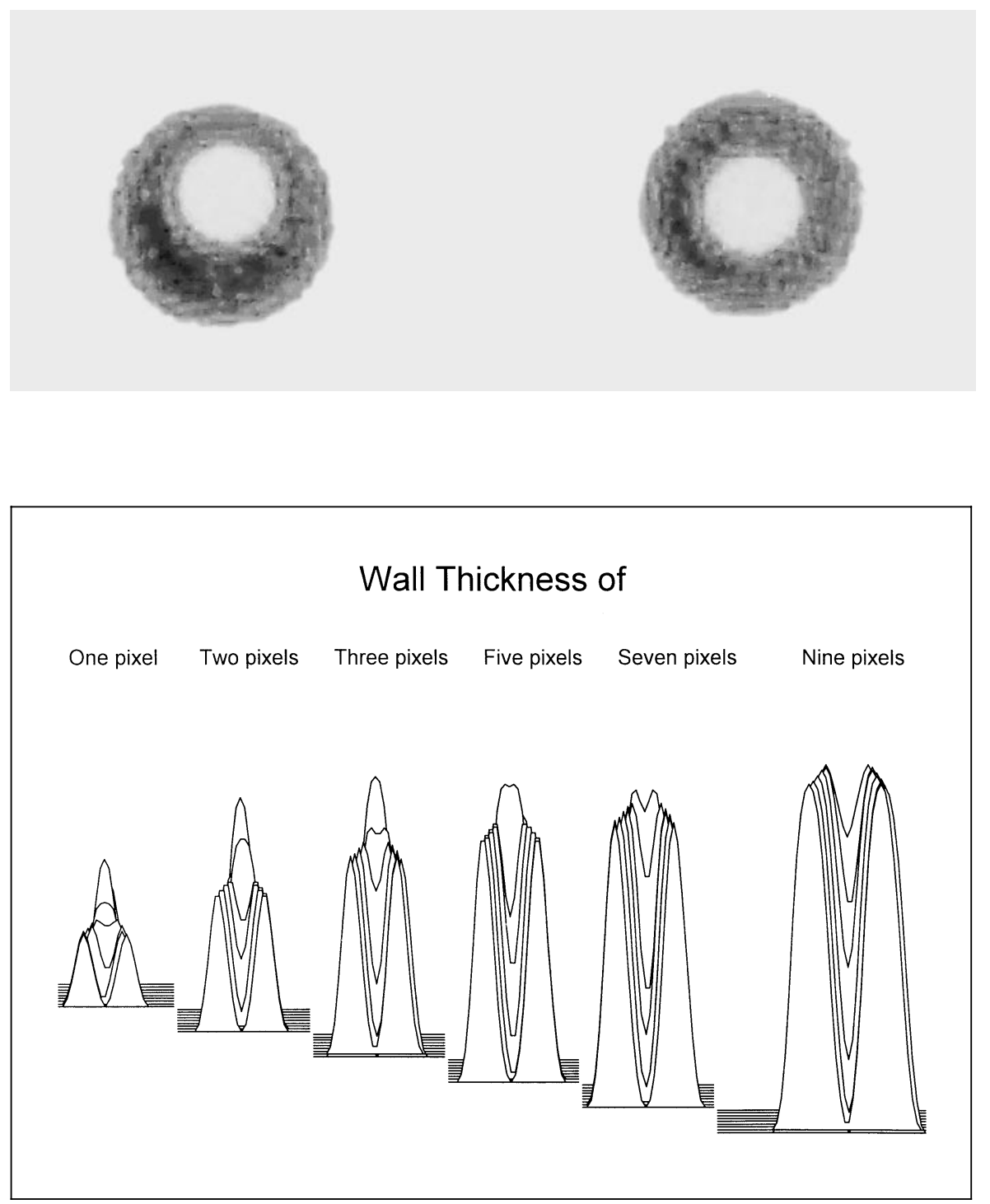

Fig. 2. Simulated image profiles. The wall thickness increases for each of the series (from left to right: 1, 2, 3, 5, 7 and 9 pixels): within each series, the separation increases (from rear to front: 1, 2, 3, 5, 7 and 9 pixels). The system resolution (FWHM) is $4.55 \pm$ 0.1 pixels. tal) were fitted to an envelope of two Gaussians to obtain estimates of the wall thickness. These measurements were obtained at apex, midheart and base. The system resolution was measured to be $\sim 18 \mathrm{~mm}$.

\section{Results}

Determination of the Spatial Resolution of the Clinical SPECT System

The system resolution (FWHM) of the SPECT system using $180^{\circ} \mathrm{C}$ acquisition was measured from the profile to be $4.55 \pm 0.1$ pixels (i.e. $18.5 \mathrm{~mm}$ ). Uncertainty in the count density was typically $\pm 5 \%$, consistent with a theoretical estimation [25].

\section{Simulation Studies}

Figure 2 shows the simulated profiles obtained by convolution of a function representing two myocardial walls of equal thickness with the experimental spatial resolution for a series of known wall thicknesses and separations. Estimates of the wall thickness obtained by fitting to two overlapping Gaussians were normalized by dividing by the FWHM of the experimental spatial resolution, and used to plot figure 3 .

For a linear spatially invariant (LSI) imaging system convolution preserves the area under a profile [26]:

$$
\mathrm{W}^{\prime} \times \mathrm{H}^{\prime}=\mathrm{W} \times \mathrm{H}
$$


Fig. 3. The estimated thicknesses (normalized by dividing by the FWHM of the system resolution) vs. the (normalized) actual thickness. The results marked $\nabla$ used fitting to a single Gaussian. All other results were estimated by fitting to two Gaussians. The separations between the walls are as follows: $\square=$ 1 pixel, $O=5$ pixels, $\triangle=9$ pixels. For clarity, not all separations are shown.

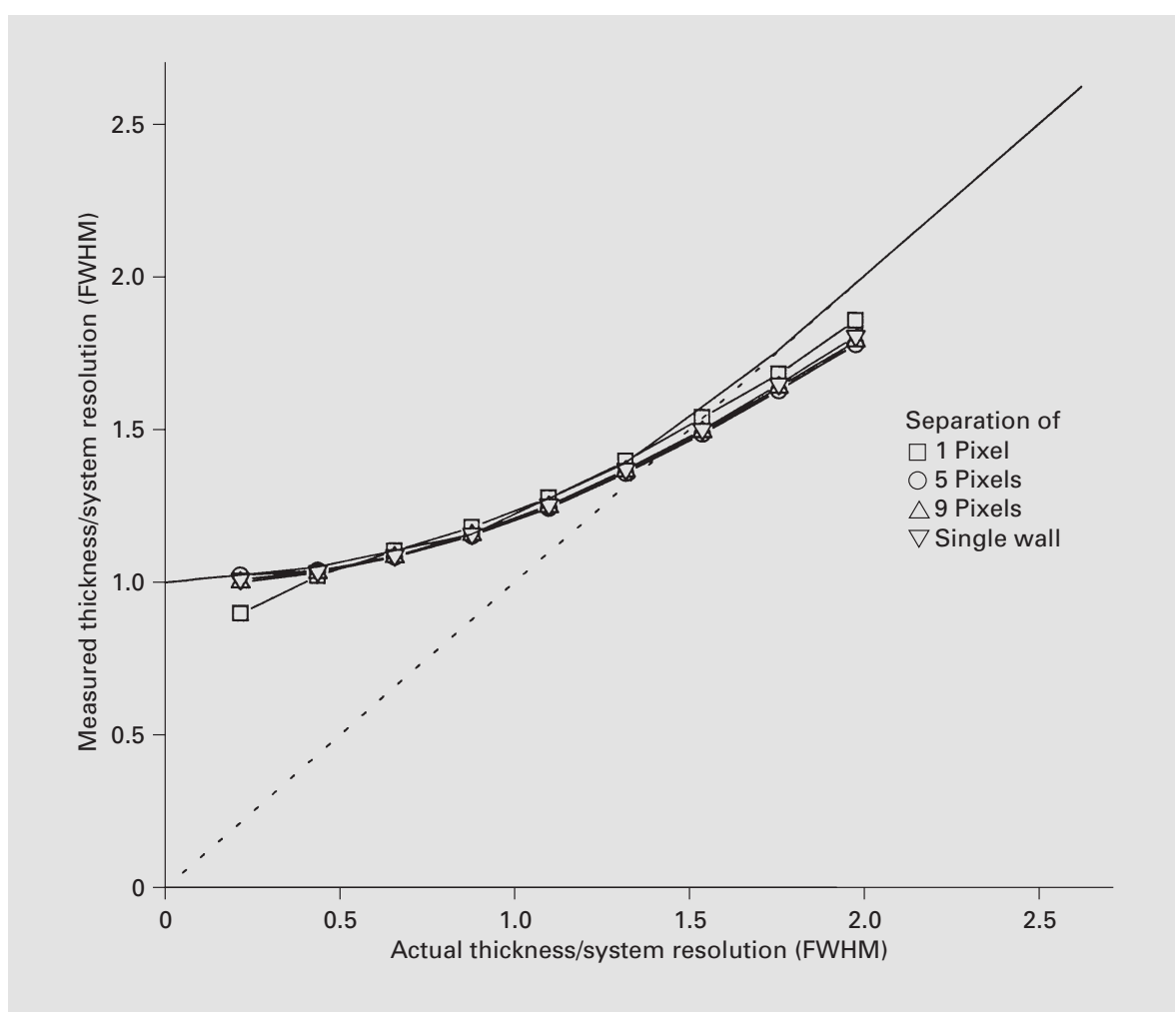

where $\mathrm{W}^{\prime}, \mathrm{W}$ are the measured and actual wall thicknesses, and $\mathrm{H}^{\prime}, \mathrm{H}$ are the measured and actual heights, respectively. Thus the ratio of actual to measured thickness from figure 3 (i.e., W/W') is equivalent to $\mathrm{H}^{\prime} / \mathrm{H}$, the ratio of measured to actual height, which was plotted to give a recovery coefficient plot as shown in figure 4 . The result is similar to that obtained in the simulation of single walls, except that the recovery coefficient rises to a value greater than unity in figure 4 at wall thicknesses larger than $1.5 \times$ FWHM (because the walls are no longer sufficiently Gaussian). Alternatively, $\mathrm{H}^{\prime}$ could be measured directly from the simulated image profiles. Once normalized by dividing by the area under the system resolution FWHM, it was plotted against the actual wall thickness (divided by FWHM) to give the conventional recovery coefficient plot (fig. 5).

Figure 6 shows different wall separations, normalized to the FWHM of the system PSF, versus the valley-topeak ratio (i.e., the ratio of the depth of the valley between two peaks to the height of the peaks representing the walls) at different thicknesses. Fitting to two overlapping Gaussians could be achieved at all valley-to-peak ratios, although the fitting at low ratios is subject to larger errors.

\section{Hot Ring Phantom}

For the hot ring phantom images estimates of the FWHMs of the two walls in each image (fig. 1) were obtained by fitting profiles to the envelope of two overlapping Gaussians (table 1): the results were independent of the separation of the walls. These estimates were then deconvolved with the system spatial resolution function using 'look-up' conversion factors from figure 3 to give 'corrected estimates' (table 1).

\section{Cardiac Phantom}

Figure 7 shows typical profiles through the ECT cardiac phantom walls at apex and mid-heart, and the results of fitting to the envelope of two Gaussians. The average (and standard deviation) of the estimates of the FWHMs of the septal, lateral, anterior, inferior, anteroseptal, anterolateral, inferoseptal and inferolateral walls, from the fitting to Gaussians, and the corrected values after deconvolution are shown in table 2 . 
Fig. 4. The ratio of actual to estimated wall thickness (using fitted Gaussians) vs. the (normalized) actual thickness. The results marked $\nabla$ used fitting to a single Gaussian. All other results were estimated by fitting to two Gaussians. The separations between the walls are as follows: $\square=1$ pixel, $O=5$ pixels, $\triangle=9$ pixels.

Fig. 5. The measured peak height of the profiles (normalized by dividing by the area under the experimental resolution) vs. the actual (normalized) wall thickness.
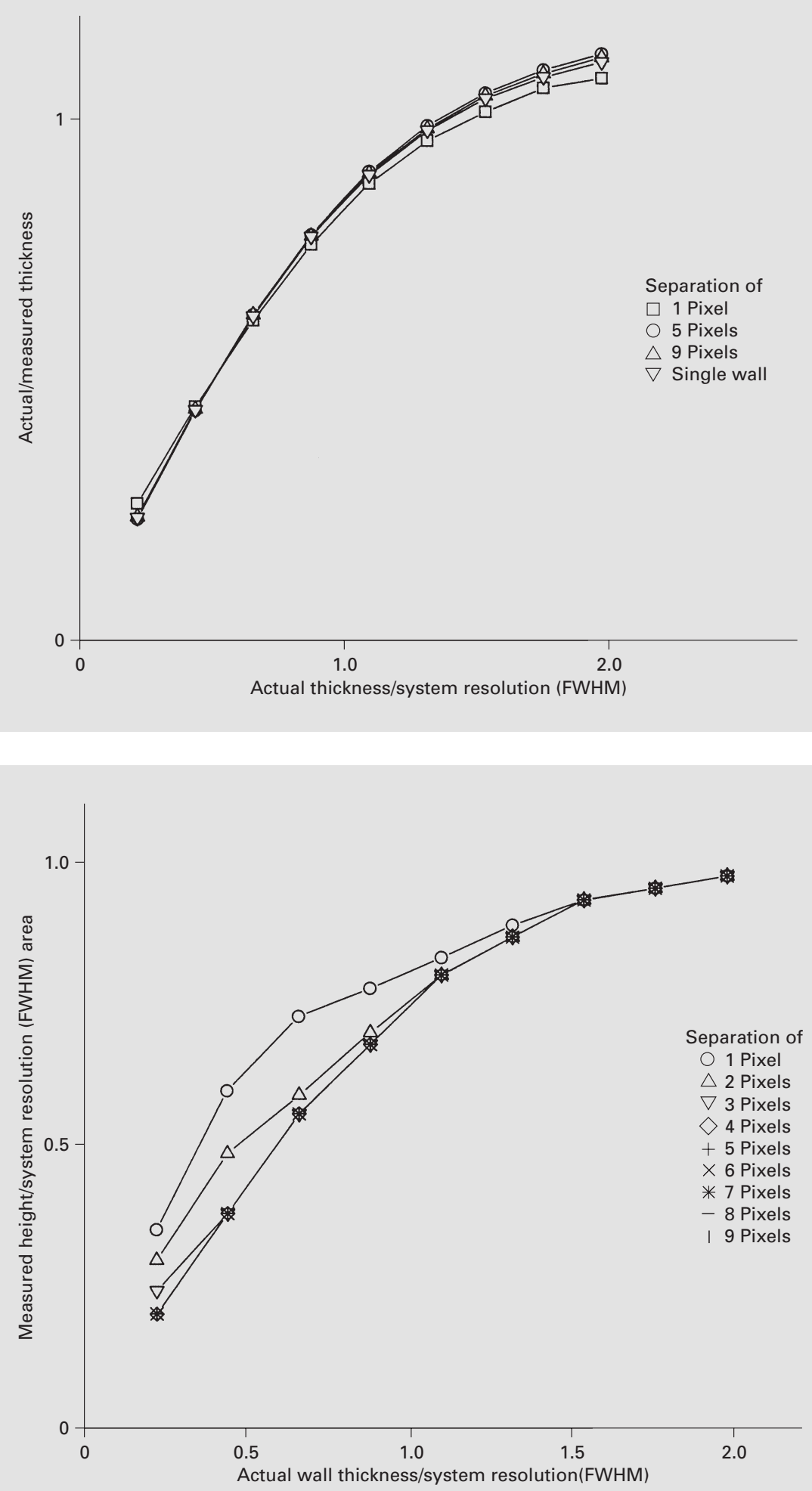
Fig. 6. Wall separation (normalized by system resolution) vs. valley-to-peak ratio. The key indicates the wall thickness.

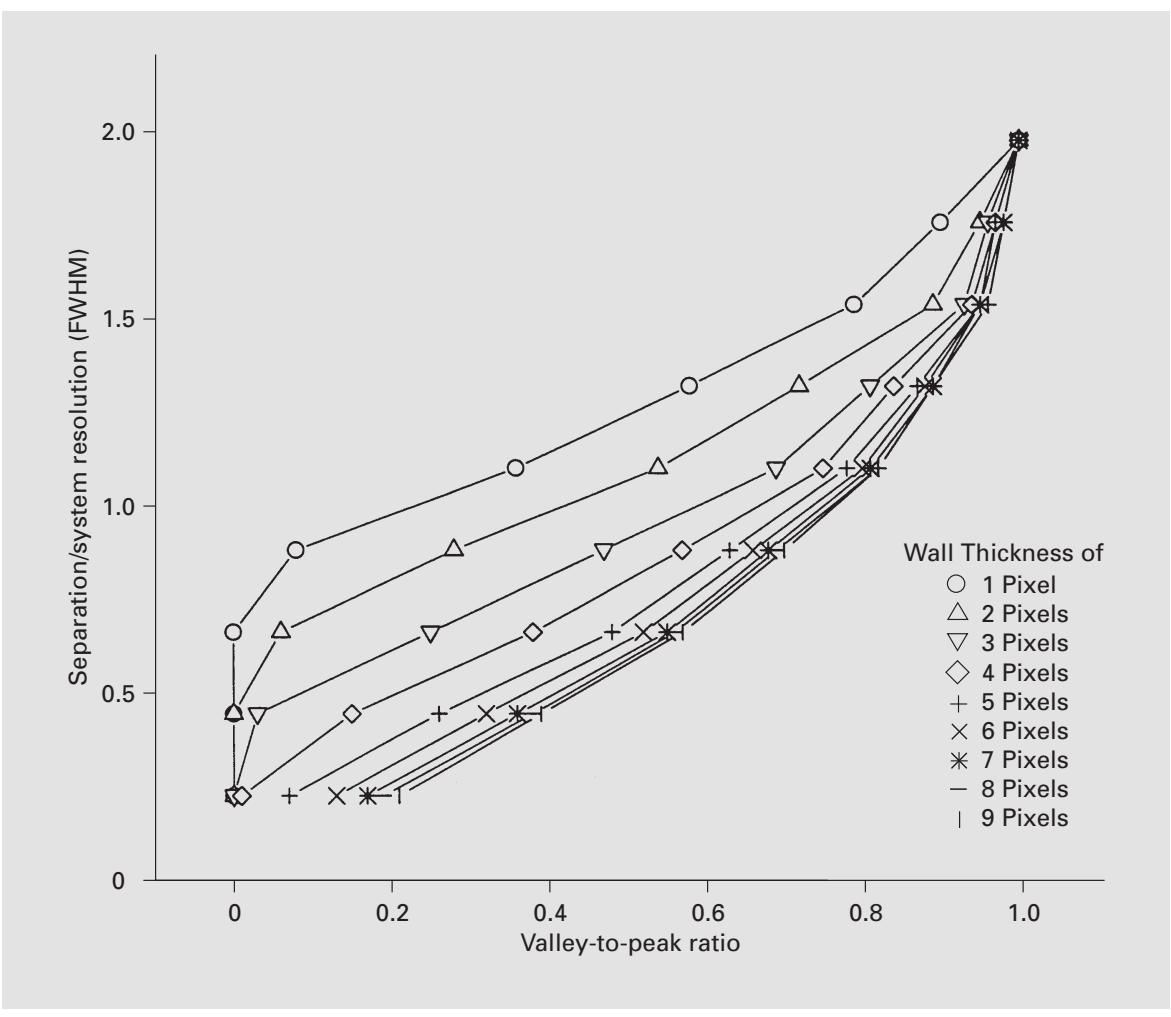

\section{Discussion}

The SPECT devices have an in-plane resolution (determined by the intrinsic resolution of the imaging device, collimator resolution, sampling intervals, reconstruction filter function, scattered radiation and raster size) different from their out-of-plane resolution (determined by slice thickness and detector resolution in the axial direction). The resulting three-dimensional PSF is not spherically symmetric. However, most studies $[1,11,15,16]$ have assumed an isotropic and stationary tomographic PSF (i.e., an LSI imaging system) with a Gaussian cross section, and relied on scaling by individual FWHMs to model the situation at a particular position in the FoV. In myocardial perfusion SPECT, acquisition over a $180^{\circ} \mathrm{C}$ axis of rotation rather than over $360^{\circ} \mathrm{C}$ is recommended [27]: the advantages are shorter acquisition time, better contrast, and in some cases better spatial resolution, at the expense of increased geometric distortion [28].

Previous studies of SPECT imaging measured wall thickening using a variety of methods, that are categorized as either count-based or geometry-based, but all of them have significant limitations. The most common count-based method relies on the percentage count in-
Table 1. Typical estimates of wall thickness from hot ring phantom images

\begin{tabular}{lll}
\hline $\begin{array}{l}\text { Wall thickness } \\
\mathrm{mm}\end{array}$ & $\begin{array}{l}\text { Estimates using } \\
\text { Gaussian fitting, } \mathrm{mm}\end{array}$ & $\begin{array}{l}\text { Corrected } \\
\text { estimates, } \mathrm{mm}\end{array}$ \\
\hline $17.0 \pm 0.3$ & $15.6 \pm 0.3$ & $17.0 \pm 0.7$ \\
$16.0 \pm 0.3$ & $14.5 \pm 0.3$ & $15.9 \pm 0.6$ \\
$25.0 \pm 0.3$ & $22.2 \pm 0.6$ & $24.0 \pm 0.7$ \\
$8.0 \pm 0.3$ & $9.9 \pm 1.1$ & $8.4 \pm 0.9$ \\
\hline
\end{tabular}

crease from end diastole to end systole as an index of thickening $[29,30]$, even though the counts are known to be underestimated in thin structures $[10,16]$. Countbased methods $[12,13,29,30]$ use only the maximum of the profile. They are very susceptible to background noise, and applying the recovery coefficient correction was cumbersome $[12,13,31]$. Geometry-based methods attempted to locate the endo- and epicardial borders by edge detection using the first [9] or second derivative [32], thresholding [1] or deconvolution [33]. The accuracy of the first derivative method depended on matrix size, noise, LSI and FWHM [9]. The second derivative method 
Fig. 7. Typical profile through the cardiac phantom walls (solid lines) at apex (O) and at mid-heart $(\square)$. The profiles were fitted to two Gaussians (dotted).

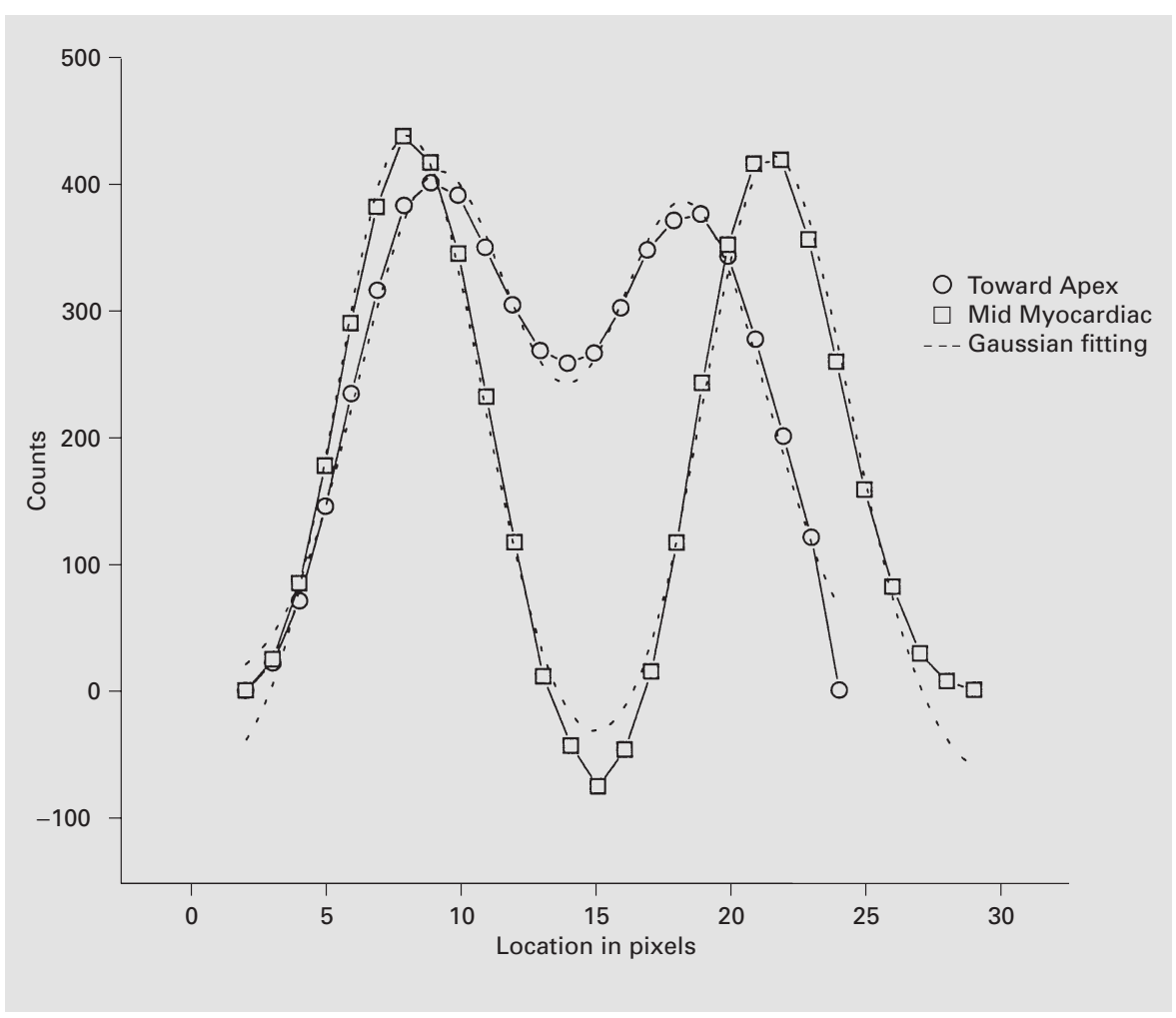

Table 2. Estimates of cardiac wall thickness in an ECT cardiac phantom using short axis slices at apex, mid- and base-heart

\begin{tabular}{|c|c|c|c|c|c|c|}
\hline & \multicolumn{2}{|l|}{ Apex } & \multicolumn{2}{|l|}{ Mid-heart } & \multicolumn{2}{|l|}{ Base-heart } \\
\hline & estimate & corrected & estimate & corrected & estimate & corrected \\
\hline Septal & $20.7 \pm 1.1$ & $12.4 \pm 0.7$ & $20.1 \pm 0.9$ & $11.6 \pm 0.6$ & $19.5 \pm 0.8$ & $10.6 \pm 0.4$ \\
\hline Lateral & $20.4 \pm 1.1$ & $12.3 \pm 0.7$ & $19.9 \pm 0.9$ & $11.5 \pm 0.6$ & $19.4 \pm 0.8$ & $10.5 \pm 0.4$ \\
\hline Anterior & $20.6 \pm 1.1$ & $12.4 \pm 0.7$ & $19.8 \pm 0.9$ & $11.5 \pm 0.6$ & $19.6 \pm 0.8$ & $10.6 \pm 0.4$ \\
\hline Inferior & $20.4 \pm 1.1$ & $11.3 \pm 0.7$ & $19.9 \pm 0.9$ & $11.5 \pm 0.6$ & $19.7 \pm 0.8$ & $10.7 \pm 0.4$ \\
\hline Anteroseptal & $20.4 \pm 1.1$ & $12.3 \pm 0.7$ & $19.7 \pm 0.9$ & $11.4 \pm 0.6$ & $19.3 \pm 0.8$ & $10.4 \pm 0.4$ \\
\hline Inferoseptal & $20.3 \pm 1.1$ & $12.3 \pm 0.7$ & $19.9 \pm 0.9$ & $11.5 \pm 0.6$ & $19.7 \pm 0.8$ & $10.7 \pm 0.4$ \\
\hline Anterolateral & $20.2 \pm 1.2$ & $12.2 \pm 0.7$ & $19.8 \pm 0.9$ & $11.5 \pm 0.6$ & $19.6 \pm 0.8$ & $10.6 \pm 0.4$ \\
\hline Inferolateral & $20.3 \pm 1.2$ & $12.3 \pm 0.7$ & $19.8 \pm 0.9$ & $11.5 \pm 0.6$ & $19.6 \pm 0.8$ & $10.6 \pm 0.4$ \\
\hline
\end{tabular}

Each estimate is the average of nine measurements: the uncertainties are $\pm 1 \mathrm{SD}$.

was dependent on the convolution matrix size and highly dependent on the object thickness [32-34]. A fixed threshold did not give the correct edges, since the required threshold was a function of the wall thickness itself $[9,23]$ and depended on the target-to-background ratio [1].
Image deconvolution was therefore unsatisfactory when the system resolution was position-dependent and its determination is adversely affected by subpixel misregistration [35]. And the hybrid method [18], which uses the integrated counts within the FWHM of the profile of each 
wall, requires complete resolution of the walls (i.e. a valley-to-peak ratio greater than 50\%) and only provides relative, not absolute, wall thickness.

Previous simulation studies of a profile comprising a single rectangular function $[1,11,14]$ showed that the measured thickness was an overestimate of the actual thickness up to about $1.75 \times$ FWHM. This was the direct result of the convolution process, and the graph relating them could be considered a look-up table that performed deconvolution. This current study showed a similar relation for a profile comprising two rectangular walls of differing widths and separation. With direct measurement (fig. 3), when it was possible (i.e., for a separation greater than $1 \times$ FWHM), the measured thickness was equal to the actual thickness above $1.75 \times$ FWHM. Direct measurement is not possible for small valley-to-peak ratios (less than 50\%), which occur for small wall thicknesses and separations (fig. 6). However, fitting the profile to two Gaussians worked well for thin walls even at small separations when they were barely resolvable or not resolvable at all. It gave an overestimate up to about $1.5 \times$ FWHM (fig. 3). Above this, the method slightly underestimated the actual thickness: this was because the convolved profile resulted from thicker walls whose images were no longer sufficiently Gaussian. There was no significant difference if the intensity (height) of the rectangular walls was changed: convolution preserved the area under the profile, and the Levenberg-Marquardt algorithm was able to fit effectively to reduced profiles.

The conventional recovery coefficient plots (fig. 5), calculated from the measured heights of the profiles, are similar in shape to the derived plots using measured thickness (fig. 4). However, the former suffered from greater measurement error since each data point was derived from a single measurement of height.

The relationship between the separation of walls (as a fraction of the system resolution) and the resulting valleyto-peak ratio (reflecting the degree to which the two walls can be resolved) is shown in figure 6 . When the separation was greater than $1.20 \pm 0.02 \times$ FWHM, the valley-topeak ratio was greater than $50 \%$ and the walls were sufficiently well resolved so that their widths (FWHM) could be measured directly from the profiles. When the separation was less than $0.65 \pm 0.03 \times$ FWHM, the valley-topeak ratio was always less than $50 \%$ and direct measurement of the walls could not be made even when the walls were thick (greater than $1.33 \times$ FWHM): fitting to Gaussians was mandatory for an accurate determination of width. At intermediate separations $(0.65-1.2 \times$ FWHM $)$, direct measurement could be used only for thicker walls, but fitting to Gaussians was applicable at all wall thicknesses.

\section{Conclusions}

The results indicate that the method produced accurate and precise values, but required more operator intervention than commercially available packages. It is a novel method of estimating wall thickness from cardiac images; this is particularly useful for estimating the width of thin poorly separated walls that are narrow compared to the PSF of the imaging system.

\section{References}

-1 Kojima A, Matsumoto M, Takahashi M, Hirota Y, Yoshida H: Effects of spatial resolution on SPECT quantification values. J Nucl Med 1989;4:508-514.

$\checkmark 2$ Hangartner TN, Battista JJ, Overton TR: Performance evaluation of density measurements of axial and peripheral bone with X-ray and gamma ray computed tomography. Phys Med Biol 1987;32:1393-1406.

>3 Flynn MJ, Cody DD: The assessment of vertebral bone macro architecture with X-ray computed tomography. Calcif Tissue Int 1993;53: S170-S175.

$\checkmark 4$ Louis O, Van den Winkle P, Covens P, Schoutens A, Osteaux M: Size of cortical bone and relationship to bone mineral density assessed by quantitative computed tomography image segmentation. Invest Radiol 1993;28:802805 .

Finite Spatial Resolution in Cardiac SPECT Imaging $\checkmark 5$ Silva MJ, Wang C, Keaveney TM, Hayes WC: Direct and computed tomography thickness measurements of the human lumbar vertebral shell and endplate. Bone 1994;15:409-414.

$\checkmark 6$ Louis O, Willnecker J, Soykens S, Van den Winkel P, Osteaux O: Cortical thickness assessed by peripheral quantitative computed tomography: Accuracy evaluated on radius specimens. Osteoporos Int 1995;5:446-449.

7 Hangartner TN, Gilsanz V: Evaluation of cortical bone by computed tomography. J Bone Miner Res 1996;11:1518-1525.

$>8$ Newman DL, Dougherty G, al Obaid A, al Hajrasy $\mathrm{H}$ : Limitations of clinical $\mathrm{CT}$ in assessing cortical thickness and density. Phys Med Biol 1998;43:619-626.

-9 Kircos LT, Carey JE, Keyes JW: Quantitative organ visualization using SPECT. J Nucl Med 1987;28:334-341.
10 Galt JR, Garcia EV, Robbins WL: Effects of myocardial wall thickness on SPECT quantification. IEEE Trans Med Imaging 1990;9:144150.

11 King MA, Long DT, Brill AB: SPECT volume quantitation: Influence of spatial resolution, source size and shape, and voxel size. Med Phys 1991;18:1016-1024.

-12 Fukuchi K, Uehara T, Morozumi T, Tsujimura E, Hasegawa S, Yutani K, Kusuoka H, Nishimura T: Quantification of systolic count increase in technetium-99m-MIBI gated myocardial SPECT. J Nucl Med 1997;38:1067-1073.

13 Germano G, Erel J, Lewin H, Kavanagh PB, Berman D: Automatic quantitation of regional myocardial wall motion and thickening from gated technetium-99m sestamibi myocardial perfusion single-photon emission computed tomography. J Am Coll Cardiol 1997;30:1360 1367. 
14 Dougherty G, Newman D: Measurement of thickness and density of thin structures by computed tomography: A simulation study. Med Phys 1999;26:1341-1348.

$\checkmark 15$ Clausen M, Bice AN, Civelek AC, Hutchus GM, Wagner HN Jr: Circumferential wall thickness measurements of the human left ventricle: Reference data for thallium-201 singlephoton emission tomography. Am J Cardiol 1986;58:527-531.

- 16 Mok DY, Bartlett ML, Bacharach SL, VoipioPulkki LM, Carson RE, Domanski MJ, Dilsizian V, Perrone-Filardi P, Bonow RO: Can partial volume effects be used to measure myocardial thickness and thickness and thickening? Comput Cardiol 1992;195-198.

$\checkmark 17$ Sechtem U, Sammerhoff BA, Markiewiag W, White RD, Cheitlin MD, Higgins CB: Regional left ventricular wall thickening by magnetic resonance imaging: Evaluation in normal persons and patients with global and regional dysfunction. Am J Cardiol 1987;59:145-151.

-18 Buvat I, Bartlett ML, Kitsiou AN, Dilsizian V, Bacharach SL: A hybrid method for measuring myocardial wall thickening from gated PET/ SPECT images. J Nucl Med 1997;38:324-329.

-19 Germano G, Kiat H, Kavanagh PB, Moriel M, Mazzanti M, Su HT, Van Train KF, Berman DS: Automatic quantification of ejection fraction from gated myocardial perfusion SPECT. J Nucl Med 1995;36:2138-2147.

-20 Faber TL, Cooke CD, Folks RD, Vansant JP, Nichols KJ, DePuey EG, Pettigrew RI, Garcia EV: Left ventricular function and perfusion from gated SPECT perfusion images: An integrated method. J Nucl Med 1999;4:650-659.
21 Faber TL, Vansant JP, Pettigrew RI, Galt JR, Blais M, Chatzimavroudis G, Cooke CD, Folks RD, Waldrop SM, Gurtler-Krawczynska E, Wittry MD, Garcia EV: Evaluation of left ventricular endocardial volumes and ejection fractions computed from gated perfusion SPECT with magnetic resonance imaging: Comparison of two methods. J Nucl Cardiol 2001;8:645651.

22 Germano G, Kavanagh PB, Waechter P, Areeda J, Van Kriekinge S, Sharir T, Lewin HC, Berman DS: A new algorithm for the quantitation of myocardial perfusion SPECT. I. Technical principles and reproducibility. J Nucl Med 2000;41:712-719.

23 Eisner RL, Paterson RE: The challenge of quantifying defect size and severity: Reality and algorithm. J Nucl Cardiol 1999;6:362371.

24 Kessler RM, Ellis JR, Eden M: Analysis of emission tomographic scan data: Limitations imposed by resolution and background. J Comput Assist Tomogr 1984;8:514-522.

25 Budinger TF, Derenzo SE, Gullberg GT, Greenberg WL, Huesman RH: Emission computer assisted tomography with single-photon and positron annihilation photon emitters. J Comput Assist Tomogr 1977;1:131-145.

26 Dougherty G: The peak CT number of profiles perpendicular to the vertebral cortical shell may be a useful indicator of the integrity of the cortical shell. Med Eng Phys 2000;22:487491.
7 Hoffman EJ: 180 degrees compared with 360 degrees sampling in SPECT. J Nucl Med 1982; 23:661-666.

28 Knesaurek K, King MA, Glick S, Penney BC: Investigation of causes of geometric distortion in 180 degrees and 360 degrees angular sampling in SPECT. J Nucl Med 1989;30:16661675 .

29 Webb S, Flower M, Ott RJ, Leach MO, Inamdar R: The spatial resolution of a rotating gamma camera tomographic facility. Br J Radiol 1983;56:939-944.

30 Long DT, King MA, Sheehan J: Comparative evaluation of image segmentation methods for volume quantitation in SPECT. Med Phys 1991;19:483-489.

31 Zito F, Gilardi MC, Magnani P, Fazio F: Single-photon emission tomographic quantification in spherical objects: Effects of object size and background. Eur J Nucl Med 1996;23: 263-271.

32 Hillel PG, Hastings DL: A three-dimensional second derivative surface-detection algorithm for volume determination on SPECT images. Phys Med Biol 1993;38:583-600.

33 Yanch JC, Irvine AT, Webb S, Flower MA: Deconvolution of emission tomographic data: A clinical evaluation. Br J Radiol 1988;61: 221-225.

34 Hillel PG, Hastings DL: The effect of phantom wall thickness on volume determination in SPET. Nucl Med Commun 1995;16:10341040.

35 Dougherty G: Effect of sub-pixel misregistration on the determination of the point spread function of a CT imaging system. Med Eng Phys 2000;22:503-507. 